\title{
Electrokinetic generation of microvortex patterns in a microchannel liquid flow
}

\author{
Alex Siu Wai Ng, Winky Lap Wing Hau, Yi-Kuen Lee \\ and Yitshak Zohar
}

Department of Mechanical Engineering, Hong Kong University of Science and Technology, Clear Water Bay, Kowloon, Hong Kong, People's Republic of China

Received 2 May 2003, in final form 27 August 2003

Published 17 November 2004

Online at stacks.iop.org/JMM/14/247 (DOI: 10.1088/0960-1317/14/2/012)

\begin{abstract}
The technology developed for micropatterning the electric surface charge to be negative, positive or neutral enables the realization of complex liquid flows in simple microchannels. A commercial CFD code is utilized to numerically simulate a variety of electrokinetically-generated liquid flows in a straight and uniform microchannel due to non-uniform surface charge distribution under an externally applied, steady electric field. We present design methodologies to electrokinetically drive vortical flows in any desired direction. In particular, we investigate surface charge patterns required to generate single or multi, co-rotating or counter-rotating, in-plane or out-of-plane vortices. Finally, in view of its potential application to microscale mixing, we discuss a surface charge pattern that can give rise to streamwise vorticity.
\end{abstract}

\section{Introduction}

Microfluidic systems have attracted major research interest due to promising potential applications in biotechnology, in particular in the lab-on-a-chip concept. Typically, an assay carried out in such a microsystem is classified into four categories: flow control, reaction, separation and detection [1-3]. Hence, microscale flow control is an integral element in the operation of a lab-on-a-chip microsystem. Pressure and gravity are traditionally the forces applied to drive liquid flows in channels. However, when the characteristic scale of the channel is too small, surface forces acting on the flow (e.g. friction along the channel solid boundaries) become very significant. Consequently, electro-osmosis driven by a surface force has recently received much attention for controlling liquid flows in microchannels [4]. Electro-osmotic flow not only becomes more efficient as the channel size becomes smaller, but also requires no moving parts. Thus, the fabrication process of microfluidic devices incorporating electro-osmosis is significantly simplified.

Most solid surfaces are likely to carry electrostatic charge, i.e. an electric surface potential, due to broken bonds and surface charge traps. When a liquid containing a small amount of ions is brought into contact with such a solid boundary, the solid-surface charge will attract the counter-ions in the liquid establishing an electric field. The arrangement of the electrostatic charges on the solid surface and the balancing charges in the liquid is called the electric double layer (EDL) [5]. Counter-ions are strongly attracted to the surface, forming an immobile compact layer at the solid/liquid interface. Outside this layer, the distribution of the counterions away from the interface decays within the diffuse double layer, the Debye layer, with a characteristic thickness inversely proportional to the square root of the liquid ion concentration. A channel with walls having fixed charges thus generates a mobile 'sheath' of charge in the fluid. Electro-osmotic flow uses an externally applied electric field parallel to a surface with net surface charge density to drive bulk motion in a fluid. The field interacts with the net charge carried by the mobile screening layer in the fluid adjacent to the surface. Under the applied electric field, the movement of this sheath transports the bulk of the fluid, as if the walls of the channel were sliding at a velocity directly proportional to both the magnitude of the applied field parallel to the walls and the electro-osmotic mobility of the charged surface. The mobility, in turn, is a function of the surface charge density, the liquid ionic strength, the liquid viscosity and permittivity.

Detailed analysis of electro-osmotic flow is very complicated, due to the non-trivial ionic distribution in the EDL, but the solution can be greatly simplified following the Debye-Hückel approximation. Under the assumptions of uniform charge distribution and uniform electric field along 
a microchannel, the resulting velocity profile resembles a plug flow. Utilizing this approximation, the effects of the EDL and surface charge on the velocity distribution and flow rate have been calculated [6]. However, theoretical studies of nonuniform charge distribution are very difficult. To date, only periodic micropatterned geometries, such as alternating strips of positive and negative surface charge, have been analyzed [7]. Numerical analysis then becomes a powerful tool to investigate electrokinetically-driven liquid flows in a microchannel having either complex geometry or complex charge distribution. Indeed, a numerical scheme has been developed to compute electro-osmotic flows in complicated geometries as in crosschannel devices for capillary electrophoresis [8]. Electroosmotic and electrophoretic transport and species diffusion have been studied by a finite difference algorithm for twodimensional complex geometry flow conduits [9]. Electroosmotic microflows in T-junctions have been studied by a finite element formulation [10] and by using meshless finite cloud algorithms [11]. Numerical calculations for pure electroosmotic and combined electro-osmotic/pressure driven Stokes flows have also been presented for the cross-flow and Y-split junctions [12]. Still, the surface charge is assumed to be uniform.

Recently, it has been recognized that complex liquid flows can also be generated by controlling the surface charge distribution. A variety of techniques have been proposed to locally generate various charge patterns. Laminar flow patterning was used to generate longitudinal patterns, while a modified version of micromolding in capillaries was used to create transverse patterns [13]. In another approach, polymer coating by an electrostatic-self-assembly (ESA) combined with standard photolithography was used to generate several different surface charge patterns [14].

In this work, a commercial CFD code is utilized to simulate liquid flows developed in a straight and uniform microchannel with a variety of complex surface charge patterns.

\section{Electrokinetically-driven liquid flow}

In a microchannel, the walls of which are made of dielectric materials, the liquid inside the channel will move in the direction of the electric field applied between an upstream and downstream location due to the viscous drag force exerted by the migrating counter-ions in the mobile diffuse region of the EDL at the solid-liquid interface. Under steady-state condition, the balance between the viscous drag force and the electro-osmotic force results in a steady flow rate with a plug-like velocity profile.

\subsection{Mathematical modeling of electro-osmotic flow}

Mathematically, electro-osmostic flow is a fluid-electrostatic coupled problem. The fluid motion in a microchannel, under an electric field, is governed by the continuity equation and the incompressible Navier-Stokes equations

$$
\begin{gathered}
\nabla \cdot \boldsymbol{v}=0 \\
\rho\left(\frac{\partial \boldsymbol{v}}{\partial t}+(\boldsymbol{v} \cdot \nabla) \boldsymbol{v}\right)=-\nabla p+\mu \nabla^{2} \boldsymbol{v}+\rho_{e} \boldsymbol{E}
\end{gathered}
$$

Here, $p$ is the pressure in $\mathrm{Pa}, \boldsymbol{v}$ is the velocity vector field in $\mathrm{m} \mathrm{s}^{-1}, \rho$ is the fluid density in $\mathrm{kg} \mathrm{m}^{-3}, \mu$ is the fluid viscosity in $\mathrm{N} \mathrm{s}^{-1} \mathrm{~m}^{-2}, \rho_{e}$ is the electric charge density in $\mathrm{C} \mathrm{m}^{-3}$, and $\boldsymbol{E}$ is the electric field intensity vector in $\mathrm{V} \mathrm{m}^{-1}$.

Under the static or quasi-static electrostatic condition, the irrotational electric field intensity is related to the electric potential $\Phi$ by

$$
E=-\nabla \Phi
$$

The electric potential is governed by the Poisson equation, taking into account the electric charge density, as follows

$$
\nabla^{2} \Phi=-\rho_{e} / \varepsilon
$$

where $\varepsilon$ is the electrical permittivity of the liquid.

The distribution of the electric charge density is governed by the distribution of the charge at the channel walls and by the externally applied electric field. The electric potential can be decomposed into two terms - the potential due to external applied electric field, $\phi$, and the potential due to the charge at the channel wall, $\psi$ - under the following conditions: (i) the Debye thickness is small compared with the channel diameter or height, (ii) the charge at the walls is not large, and (iii) the fluid velocity in the microchannel is too small to redistribute the electric charge distribution (the typical Reynolds number for electro-osmotic flow is less than 0.01). Equation (4) can now be split into the following two equations:

$$
\begin{gathered}
\nabla^{2} \phi=0 \\
\nabla^{2} \psi=-\rho_{e} / \varepsilon .
\end{gathered}
$$

Utilizing the charge distribution proposed in Gouy-Chapman theory [15], the EDL is modeled as a diffuse layer, which consists of a series of laminae parallel to the microchannel wall. All the ions in these laminae are assumed to be in thermal equilibrium. Therefore, the ion distribution satisfies Boltzmann statistics. The electric potential at a fixed distance away from the channel wall is assumed to be a constant zeta potential. Therefore, equation (6) can be simplified to

$$
\nabla^{2} \psi=\kappa^{2} \psi
$$

where $\kappa^{-1}\left(=\lambda_{D}\right)$ is the Debye length. For aqueous solutions at $25^{\circ} \mathrm{C}$, ionic concentrations of $10^{-5} \mathrm{M}$ and $10^{-3} \mathrm{M}$ correspond to the Debye lengths of 100 and $10 \mathrm{~nm}$, respectively.

It is important to note that there are many theories describing the EDL. Although the Gouy-Chapman theory includes questionable assumptions and more complex theories have evolved, nonetheless the Gouy-Chapman theory provides an often-used convenient model to describe the EDL.

\subsection{Electro-osmotic flow in a straight and uniform microchannel}

A commercial CFD code (CFDRC, USA) is used to simulate the electro-osmotic flows investigated in this work. All the simulations are carried out for water as the working liquid having density $\rho=10^{3} \mathrm{~kg} \mathrm{~m}^{-3}$, permittivity $\varepsilon=6.95 \times$ $10^{-10} \mathrm{C}^{2} \mathrm{~J}^{-1} \mathrm{~m}^{-1}$ and viscosity $\mu=10^{-3} \mathrm{~N} \mathrm{~s}^{-1} \mathrm{~m}^{-2}$. The Debye length scale and zeta potential are fixed at $\lambda_{D}=$ $0.1 \mu \mathrm{m}$ and $\zeta= \pm 25 \mathrm{mV}$, respectively, while the electric field strength is selected to be $E=3.3 \mathrm{~V} \mathrm{~mm}^{-1}$. The microchannel top and bottom plates are placed at $y= \pm H / 2=25 \mu \mathrm{m}$ and 


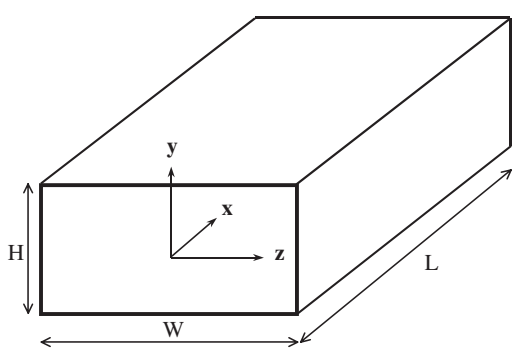

(a)

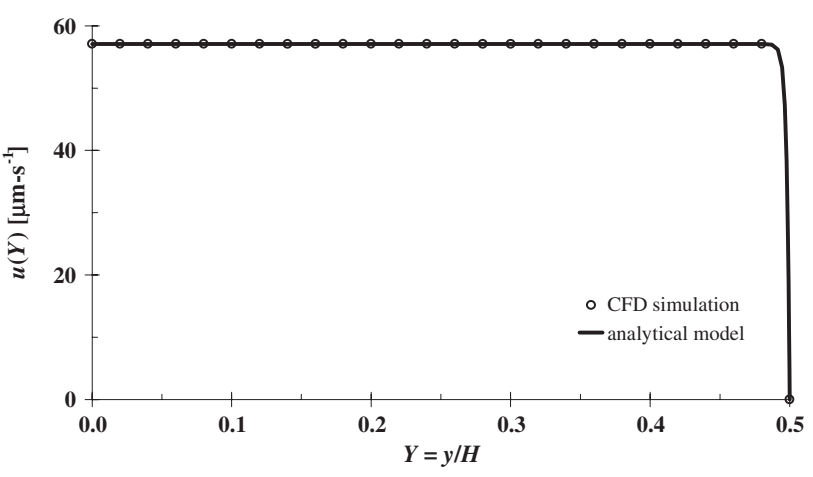

(b)

Figure 1. A comparison between CFD results and theoretical calculations: $(a)$ the physical model of a rectangular microchannel; (b) the velocity profile across a $2 \mathrm{D}$ microchannel, $W \rightarrow \infty$, with a uniform surface charge under a uniform electric field.

the sidewalls are located at $z= \pm W / 2=50 \mu \mathrm{m}$, with the microchannel inlet set at $x=0$ and the outlet at $x=L=$ $1050 \mu \mathrm{m}$ as schematically illustrated in figure 1(a).

The validity of the code is first verified by examining the streamwise velocity profile of the pure electro-osmotic flow developed between two infinite plates, separated by a small gap $H$, for which a closed-form analytical solution exists. The twodimensional (2D) solution of the streamwise velocity profile along the microchannel $x$-direction, for small zeta potential and much smaller EDL thickness compared to the gap height, is given by [8]

$$
u(Y)=\frac{E \varepsilon \zeta}{\mu}\left[\frac{\cosh (Y / \lambda)}{\cosh (1 / \lambda)}-1\right]
$$

where $\lambda\left(=\lambda_{D} / h\right)$ is the normalized Debye length, $Y(=y / h)$ is the normalized cross-stream coordinate $y$ with the origin at mid-plane, and $h(=H / 2)$ is the channel half-height. The calculated cross-stream distribution of the streamwise velocity component, based on equation (8), is plotted in figure $1(b)$. To benchmark the CFD code, a 2D numerical model is constructed assuming $W \rightarrow \infty$ with the top and bottom surfaces carrying uniform charge. The computed velocity profile is compared with the analytical one in figure $1(b)$, where only half of the profile is shown due to the symmetry of the flow. The agreement between the theoretical and numerical electroosmotic velocity of about $57 \mu \mathrm{m} \mathrm{s}^{-1}$ is satisfactory.

Strong velocity gradients are expected not only near the walls, due to the EDL, but also in the flow interior, due to the patterned surface charge. Hence, a uniform mesh is adopted. The effect of the mesh size on the accuracy of the solution has been tested, and the results are summarized in figure 2 . The three-dimensional (3D) solution for the flow in the

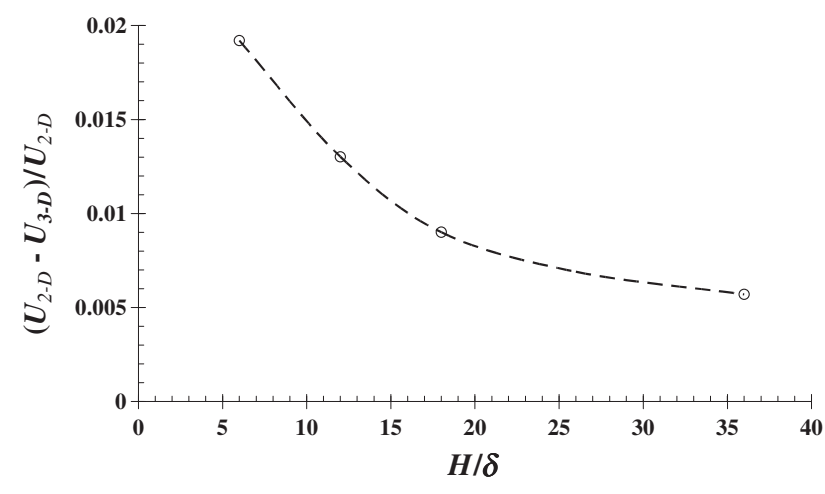

Figure 2. Convergence of the electro-osmotic velocity in a microchannel, having a uniform negative surface charge with zeta potential of $\zeta=-25 \mathrm{mV}$ and under an electric field of $E=$ $3.3 \mathrm{~V} \mathrm{~mm}^{-1}$, as a function of the distance between grid points $\delta$.

$100 \mu \mathrm{m}$ wide microchannel, $U_{3 \mathrm{D}}$, converges to less than $1 \%$ of the theoretical value $U_{2 \mathrm{D}}$, calculated from equation (8), when using more than 15 grid points across the channel height. Thus, the employed CFD package with uniform mesh is accurate enough for computations of electro-osmotic flows with small zeta potential.

\section{Vortex flow patterns}

The development of the surface-chemistry technology to control the surface charge distribution to be positive, negative or neutral has enabled the generation of a large class of complex liquid flows in straight and uniform microchannels. In particular, design methodologies are presented to create vortical flows, driven by a single electric field along the microchannel, with vorticity component in any desired direction.

\subsection{Cross-stream vorticity dynamics $\left(\Omega_{y}\right)$}

Under an externally applied streamwise electric field in the $x$-direction as shown in figure $3(a)$, the resulting electroosmotic flow is usually in the streamwise direction as well. In order to generate flows with cross-stream vorticity component, defined as $\Omega_{y} \equiv \partial u / \partial z-\partial w / \partial x$, a non-uniform spanwise surface charge distribution is required to induce the spanwise velocity component. The combination of spatially nonuniform streamwise and/or spanwise velocity components, i.e. $u(z)$ and $w(x)$, can result in in-plane rotational motion giving rise to cross-stream vorticity.

A simple heterogeneous configuration to create a single in-plane vortex, as first sketched by Ajdari [7], is illustrated in figure 3(a) with $W_{a}=L_{a}=50 \mu \mathrm{m}$. A simple charge pattern of a positively charged zone next, in the spanwise direction, to a negatively charged zone is placed at equal distance from the channel inlet and outlet. The top and bottom surfaces are symmetrically charged, while the rest of the microchannel surface is neutral. The electro-osmotic slip generated by the streamwise electric field leads to a recirculation zone. The flow field for a given set of values has been numerically calculated. The resulting velocity vector at the channel mid-plane, parallel to the top and bottom surfaces, is plotted in figure $3(b)$. A 


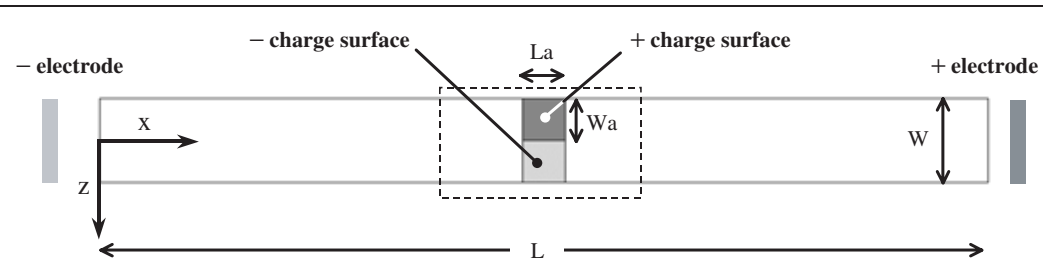

(a)

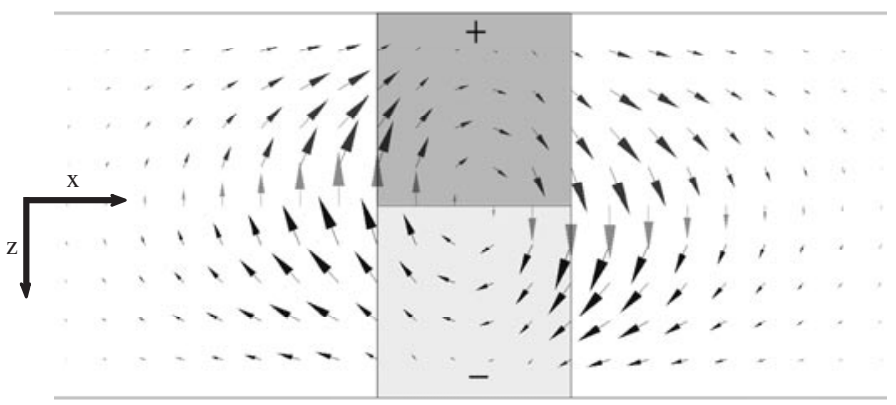

(b)

Figure 3. A single in-plane microvortex: (a) planar view of the top/bottom surface-charge pattern; $(b)$ the corresponding velocity field at mid-plane, $y=0$.

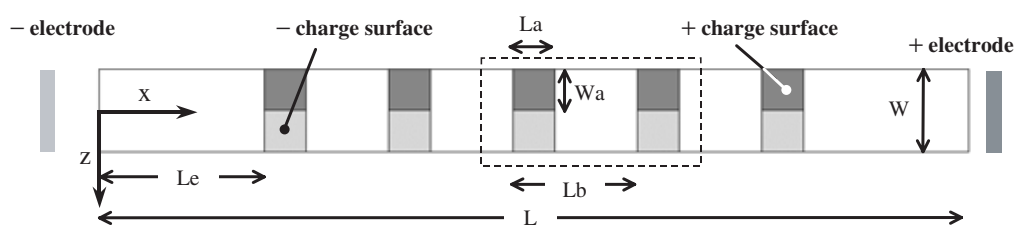

(a)

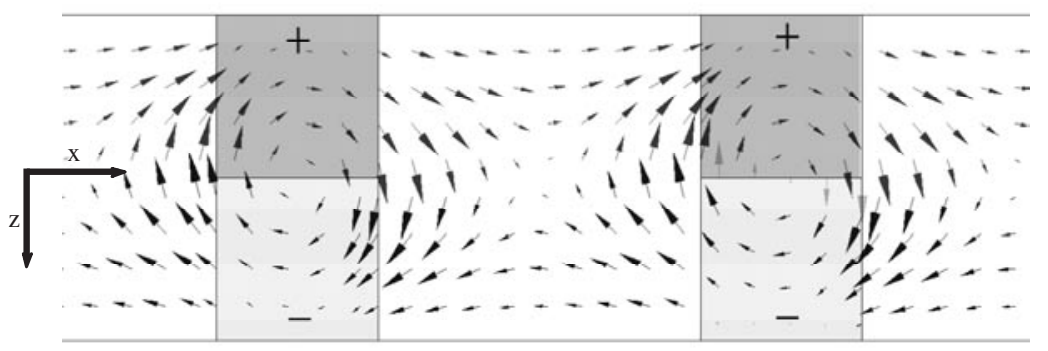

(b)

Figure 4. A train of in-plane co-rotating vortices: (a) planar view of the top/bottom surface-charge pattern; $(b)$ the corresponding velocity field at mid-plane, $y=0$, for $L_{a} / L_{b}=1 / 3$.

single in-plane vortex is obtained rotating clockwise. Clearly, either reversing the streamwise electric field or flipping the positive/negative charge pattern will reverse the vortex flow to counter-clockwise direction.

Streamwise periodic duplication of the charge pattern required for a single vortex is schematically drawn in figure $4(a) . L_{a}$ is the length of the active surface charge and $L_{b}$ is the length of the periodic cell, with five cells along the microchannel. The first and last cells are located at a distance $L_{e}=200 \mu \mathrm{m}$ far from the channel inlet and outlet to minimize edge effects. The corresponding flow field has been numerically simulated for $L_{b}=3 L_{a}=150 \mu \mathrm{m}$, and the resulting velocity vector plot at the channel mid-plane is shown in figure $4(b)$. A train of five in-plane co-rotating vortices is produced, of which two are plotted. The wavelength of this periodic vortical pattern is equal to $L_{b}$, where all vortices rotate in the clockwise direction. Again, either reversal of the electric field or flipping the charge pattern will result in reversal of the rotational direction of the vortex train.

In the limiting case of $L_{a} \rightarrow L_{b}$, all the cells merge together, dividing the channel bottom and top surfaces into two halves; one half is negatively charged while the other is positively charged. The calculated velocity vector field for this case is depicted in figure 5. A bi-directional shear flow is formed with velocity gradient in the spanwise direction, $u(z)$, while in the cross-stream direction the velocity field resembles plug flow.

It is also possible to generate a periodic flow of counterrotating pairs of in-plane vortices. This can be done by flipping the charge pattern in every alternate cell in the configuration used for the co-rotating vortices, as illustrated in figure $6(a)$. The wavelength of the corresponding velocity vector field, 


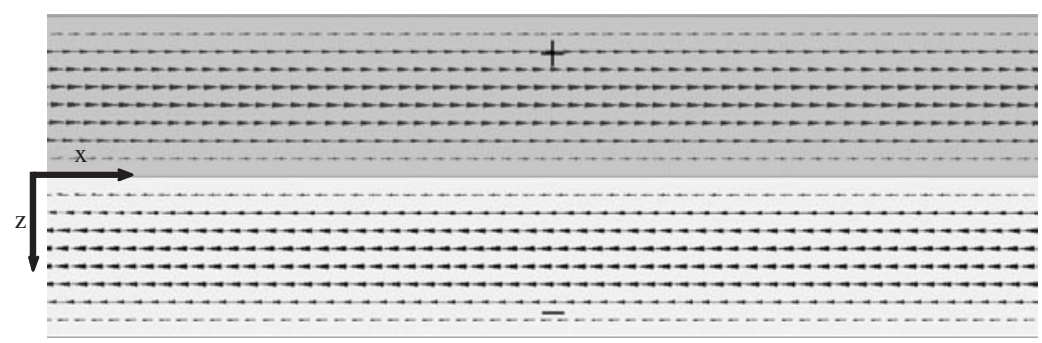

Figure 5. Top view of the pure bi-directional shear flow along the microchannel side walls at mid-plane, $y=0$, as a limit case of vanishing neutral surface charge regions between the periodic active cells shown in figure $3\left(L_{a} / L_{b}=1\right)$.

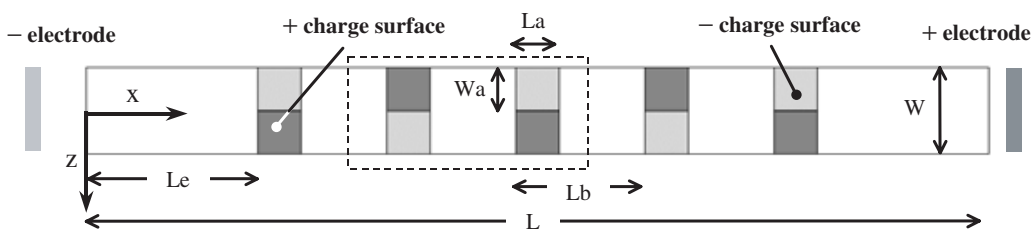

(a)

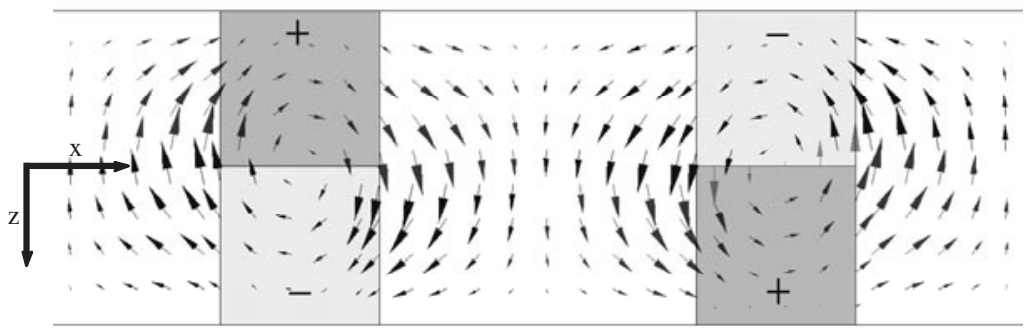

(b)

Figure 6. A pair of in-plane counter-rotating vortices: $(a)$ planar view of the top/bottom surface-charge pattern; $(b)$ the corresponding velocity field at mid-plane, $y=0$, for $L_{a} / L_{b}=1 / 3$.

shown in figure $6(b)$, has doubled to $2 L_{b}=300 \mu \mathrm{m}$. Similar flow pattern was experimentally realized for the case of vanishing neutral zones between the surface-charged regions [16].

These in-plane vortical flow fields can be summarized using the cross-stream vorticity component. The computed streamwise distributions of the spanwise velocity, $w(x)$, and cross-stream vorticity, $\Omega_{y}(x)$, along the microchannel at the channel mid-height $y=0$ are plotted in figure 7 . The single cell velocity profile shown in figure $7(a)$ is typical of a viscous vortex, antisymmetric with respect to the vortex core at $x / L=$ 0.5 , with a maximum velocity of about $30 \mu \mathrm{m} \mathrm{s}^{-1}$. The velocity profile for the co-rotating charge pattern $\left(L_{a} / L_{b}=\right.$ $1 / 3$ ) comprises five similar cycles, corresponding to the five cells, with the same amplitude as for the single vortex. The vorticity distribution for the single vortex (filled circular symbols), shown in figure 7(b), contains only one peak of about $-2.5 \mathrm{~s}^{-1}$ at the channel center $x / L=0.5$. For the co-rotating case $\left(L_{a} / L_{b}=1 / 3\right.$; dashed line), there are five peaks corresponding to the five cells illustrated in figure 4 . The vorticity between the peaks drops from its maximum of about $-2.5 \mathrm{~s}^{-1}$ to zero indicating no interaction between the individual cells. However, to illustrate the effect of varying the ratio $L_{a} / L_{b}$ in these periodic charge patterns, the results for the case of $L_{a} / L_{b}=0.8$ are also included. As the ratio $L_{a} / L_{b}$ gradually approaches unity, the vorticity between the periodic vortex cores increases while the peak vorticity remains about the same $\left(-2.5 \mathrm{~s}^{-1}\right)$. At the limit $L_{a} / L_{b}=1$, the streamwise distribution of the cross-stream vorticity becomes uniform at the peak level of $\Omega_{y}(x)=-2.5 \mathrm{~s}^{-1}$ (solid line), corresponding to a pure shear flow. The counter-rotating vortices (hollow circular symbols), illustrated in figure 6 , exhibit three positive and two negative lobes with identical absolute vorticity level $\left|\Omega_{y}(x)\right|=2.5 \mathrm{~s}^{-1}$.

\subsection{Spanwise vorticity dynamics $\left(\Omega_{z}\right)$}

The idea of generating flows with cross-stream vorticity can be extended to generate flows with the spanwise vorticity component, defined as $\Omega_{z} \equiv \partial v / \partial x-\partial u / \partial y$, under a single streamwise electric field. The simplest approach is to pattern the surface charge of both sidewalls symmetrically in the exact same configurations used for the top and bottom surfaces to generate the cross-stream vorticity $\Omega_{y}$. In such a case, all the flows discussed in section 3.1 can be duplicated with $90^{\circ}$ rotation with respect to the channel axial direction $x$. Although mathematically correct, technologically it is very difficult to pattern the channel sidewalls since photolithographic pattern transfer usually requires a planar surface. Furthermore, in most microchannel applications, the channel width is typically much larger that its height. Therefore, patterning the sidewalls to generate spanwise vortical flows is not a practical option.

However, in order to generate spanwise vorticity, it is sufficient to induce non-uniform cross-stream distribution of the streamwise velocity component, i.e. $u(y)$. This can be achieved rather easily by patterning the top and bottom 


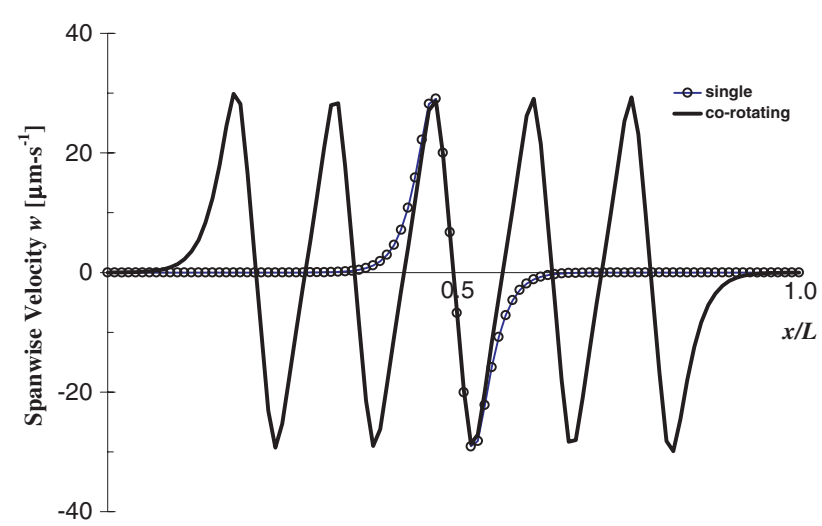

(a)

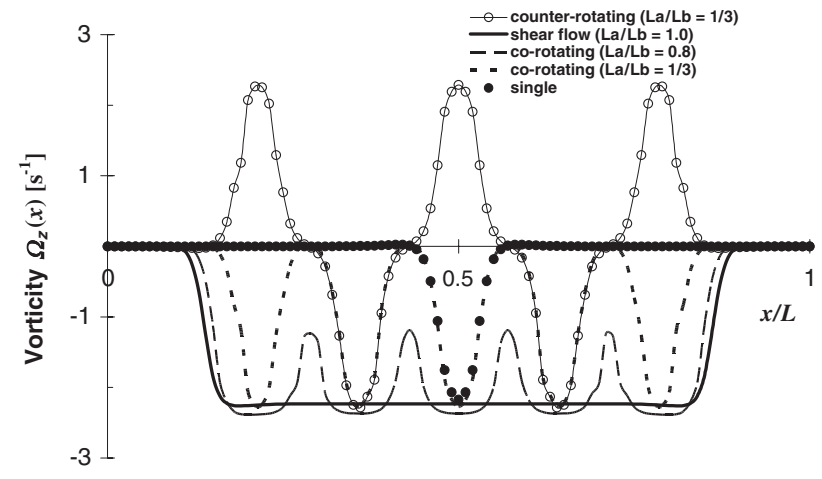

(b)

Figure 7. Streamwise distributions along the microchannel centerline $(y=z=0)$ of $(a)$ spanwise velocity, $w(x)$, and $(b)$ cross-stream vorticity, $\Omega_{y}(x)$, for the in-plane vortical flow fields.

surfaces antisymmetrically. Under a streamwise electric field, the liquid near the top and bottom surfaces will flow in opposite directions giving rise to spanwise vorticity. Following this approach all the previous flow fields can be created by patterning the surface charge of the top and bottom surfaces only while keeping the sidewalls neutral.
The surface-charge pattern for generating a single out-ofplane spanwise vortex, shown in figure $8(a)$, is even more simple than that used in forming the in-plane vortex. A positively or negatively charged zone is required on either the top or bottom surface, $W_{a}=2 L_{a}=100 \mu \mathrm{m}$, while the corresponding zone on the opposite surface carries the opposite charge. The active area is placed at the center, while the rest of the microchannel surface is kept neutral. The opposite electro-osmotic slip, generated by a streamwise electric field, near the top and bottom surfaces leads to a recirculating zone. The numerically simulated velocity field for this surfacecharge configuration is plotted in figure $8(b)$. A single out-of-plane vortex is clearly visible rotating clockwise around its axis in the spanwise $z$-direction. Again, to obtain counter-clockwise vortex flow direction, either the streamwise electric field or the surface charge pattern has to be reversed.

A train of co-rotating out-of-plane vortices can be formed by periodic duplication of the charge pattern required for a single vortex as schematically drawn in figure $9(a)$ for five such cells. The flow field has been calculated numerically for $L_{b}=3 L_{a}=150 \mu \mathrm{m}$, with $L_{e}=2 W_{a}=200 \mu \mathrm{m}$, and the corresponding velocity vector plot at the channel mid-plane is shown in figure $9(b)$. A series of five out-of-plane co-rotating vortices develop under the applied electric field, of which two are plotted. The wavelength of the periodic vortical pattern is equal to $L_{b}$ with clockwise rotation. Either the reversal of the electric field or flipping the charge pattern between the top and bottom surfaces will result in reversing the rotational direction of the vortex train.

In the limiting case of $L_{a} \rightarrow L_{b}$, positive charge is uniformly distributed on the top surface while negative charge is uniformly distributed on the bottom surface. The calculated velocity vector field is depicted in figure 10. A pure shear flow is formed with velocity gradient in the cross-stream direction, $u(y)$, reminiscent of the classical Couette flow. The flow field is almost $2 \mathrm{D}$, except near the two sidewalls due to the no-slip boundary condition.

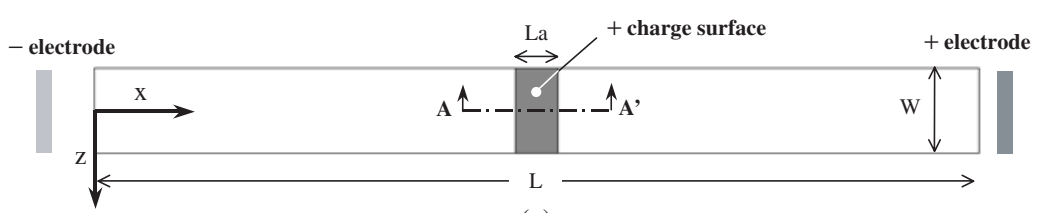

(a)

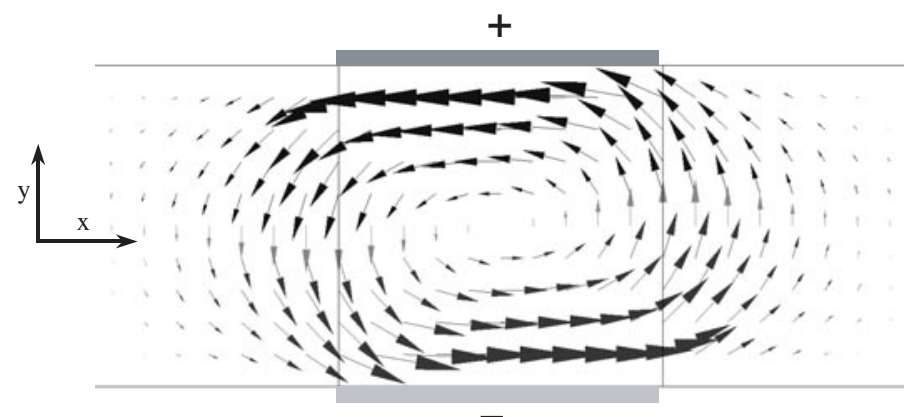

(b)

Figure 8. A single out-of-plane microvortex: $(a)$ planar view of the top surface-charge pattern; $(b)$ side view of the corresponding velocity field at central plane, $z=0$. 


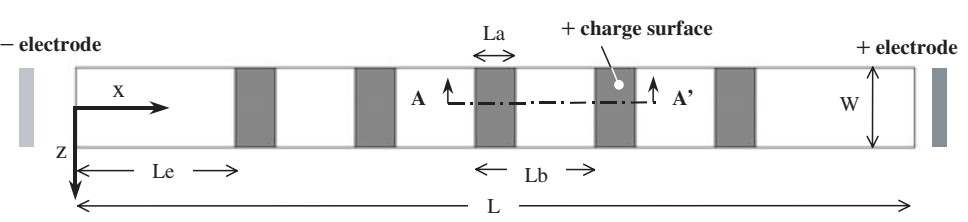

(a)

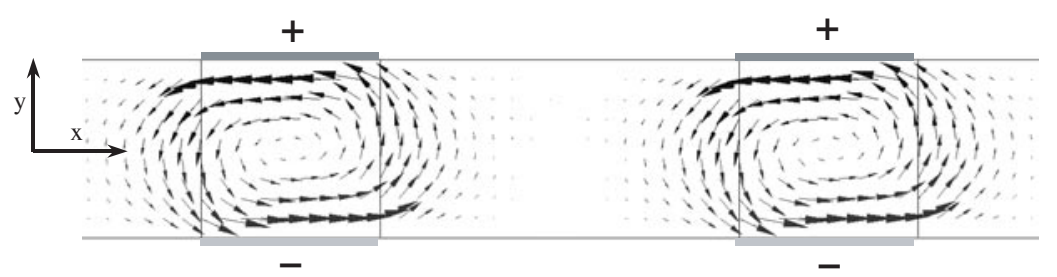

(b)

Figure 9. A train of out-of-plane co-rotating vortices: $(a)$ planar view of the top surface-charge pattern; $(b)$ side view of the corresponding velocity field at central plane, $z=0$, for $L_{a} / L_{b}=1 / 3$.

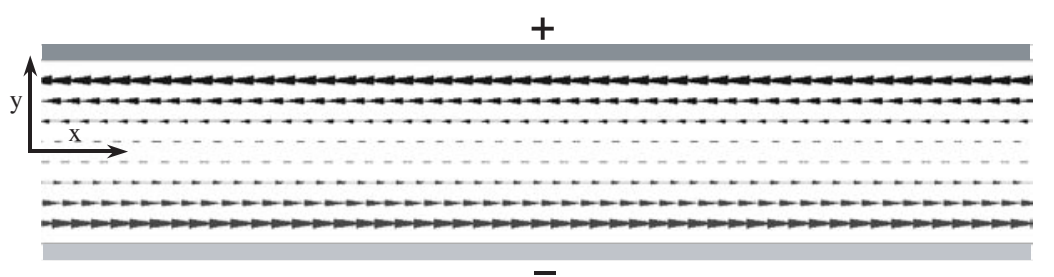

Figure 10. Side view of the pure bi-directional shear flow along the microchannel top/bottom walls at central plane, $z=0$, as a limit case of vanishing neutral surface charge regions between the periodic active cells shown in figure $8\left(L_{a} / L_{b}=1\right)$.

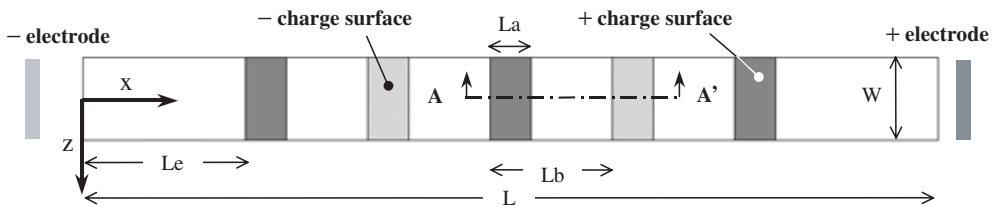

(a)

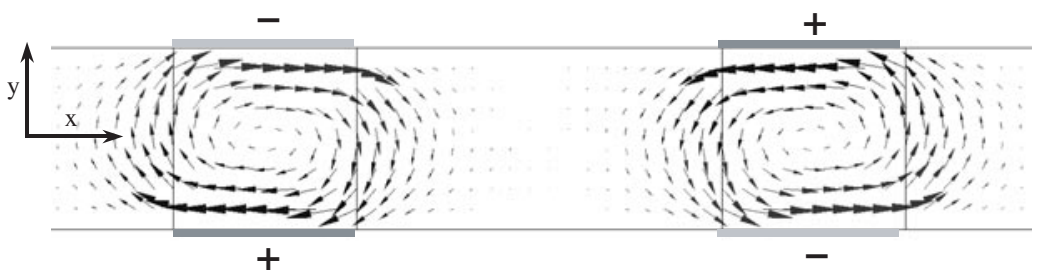

(b)

Figure 11. A pair of out-of-plane counter-rotating vortices: $(a)$ planar view of the top surface-charge pattern; $(b)$ side view of the corresponding velocity field at central plane, $z=0$, for $L_{a} / L_{b}=1 / 3$.

Counter-rotating pairs of out-of-plane vortices periodically distributed along the channel can be formed by flipping the charge pattern in every alternate cell in the configuration used for the co-rotating vortices, as illustrated in figure 11 $(a)$. The wavelength of the corresponding velocity vector field, depicted in figure $11(b)$, has doubled to $2 L_{b}=$ $300 \mu \mathrm{m}$. A similar vortex flow field was experimentally demonstrated for the particular case of vanishing neutral zones between the surface-charged regions [17].

The out-of plane vortical flow fields are summarized using the spanwise vorticity component. The computed streamwise distributions of the cross-stream velocity, $w(x)$, and spanwise vorticity, $\Omega_{z}(x)$, along the microchannel at the channel mid-height $y=0$ are plotted in figure 12. An antisymmetric velocity profile indicating a viscous vortex at $x / L=0.5$ for the single-cell pattern is evident in figure $12(a)$, with a maximum of about $20 \mu \mathrm{m} \mathrm{s}^{-1}$. The velocity profile for the counterrotating charge pattern $\left(L_{a} / L_{b}=1 / 3\right)$ comprises five positive and five negative peaks, corresponding to the five cells, with the same amplitude as for the single vortex. However, the velocity profile in every alternate cell is horizontally flipped doubling the wavelength since each periodic cycle in this case contains a pair of counter-rotating vortices. Measurements of peak velocities of the order of $50 \mu \mathrm{m} \mathrm{s}^{-1}$ have been reported in similarly patterned electro-osmotic flow [13]. The vorticity distribution for the single vortex (filled circular symbols), 


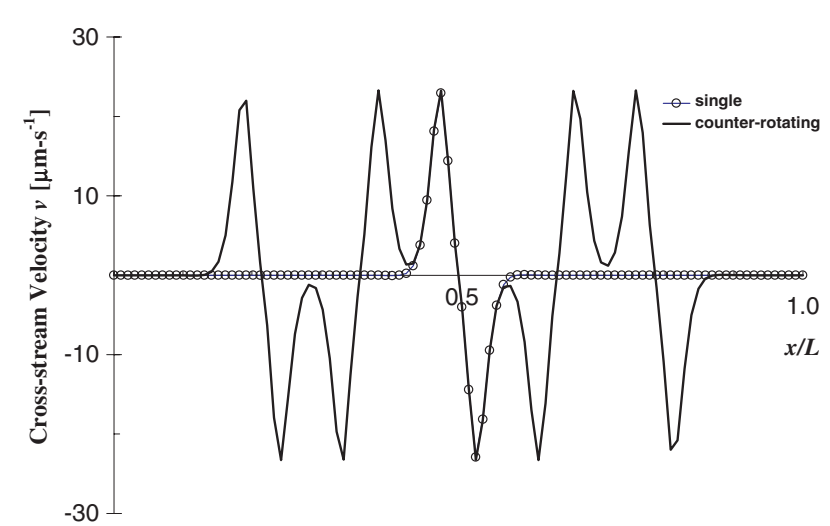

(a)

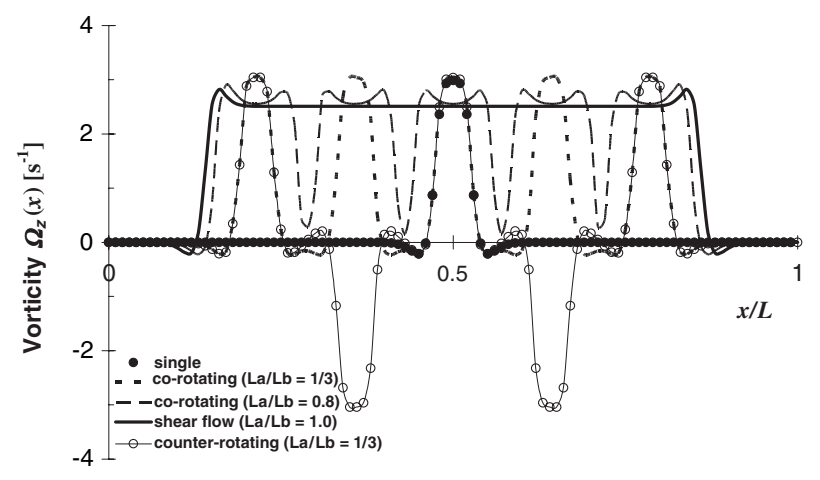

(b)

Figure 12. Streamwise distributions along the microchannel centerline $(y=z=0)$ of ( $a$ ) cross-stream velocity, $v(x)$, and $(b)$ spanwise vorticity, $\Omega_{z}(x)$, for the out-of-plane vortical flow fields.

shown in figure $12(b)$, contains only one peak of about $3 \mathrm{~s}^{-1}$ at the channel center $x / L=0.5$. For the co-rotating case $\left(L_{a} / L_{b}=1 / 3\right.$; dashed line), there are five peaks at the same level of about $3 \mathrm{~s}^{-1}$ corresponding to the five cells illustrated in figure 9 . The vorticity between the peaks drops below zero indicating a weak interaction between the individual cells. To illustrate the effect of varying the ratio $L_{a} / L_{b}$ in these periodic charge patterns, the simulation results for the case of $L_{a} / L_{b}=$ 0.8 are also included. As the ratio $L_{a} / L_{b}$ gradually approaches unity, the vorticity between the periodic vortex cores increases while the peak vorticity decreases from 3 to about $2.5 \mathrm{~s}^{-1}$. At the limit $L_{a} / L_{b}=1$, the streamwise distribution of the spanwise vorticity becomes uniform at the level of $\Omega_{z}(x)=2.5 \mathrm{~s}^{-1}$ (solid line), corresponding to a pure shear flow. The out-of-plane counter-rotating vortices (open circles), illustrated in figure 11, exhibit three positive and two negative lobes with identical absolute vorticity level $\left|\Omega_{y}(x)\right|=3 \mathrm{~s}^{-1}$.

\subsection{Streamwise vorticity dynamics $\left(\Omega_{x}\right)$}

It is not easy to generate simple and orderly patterns of streamwise vorticity, defined as $\Omega_{x}=\partial w / \partial y-\partial v / \partial z$. Externally applied streamwise electric field induces only the streamwise velocity component, while streamwise vorticity requires gradients in the spanwise or cross-stream velocity component. However, combining the approaches for generating the in-plane and out-of-plane vortices, with neutral sidewalls, streamwise vorticity can also be generated. Applying only a streamwise electric field across a channel having a chessboard-like charge pattern illustrated in figure 13(a), with antisymmetric distributions on the top and bottom surfaces, can generate both velocity components. The microchannel width is doubled to $W=200 \mu \mathrm{m}$ while the dimensions of each charged region are $L_{a}=W_{a}=50 \mu \mathrm{m}$. The entire chessboard pattern is placed at the channel center far from the microchannel inlet/outlet. The calculated velocity field was used to obtain the vorticity distribution, and contours of constant streamwise vorticity $\Omega_{x}(y, z)$ at $\mathrm{A}-\mathrm{A}^{\prime}$ channel cross-section are shown in figure $13(b)$. Indeed, the existence of coherent streamwise vortical eddies is evident.

This class of flow fields, where vorticity in all directions can be generated, is in particular important for mixing of liquids in microsystems. Preliminary results indeed

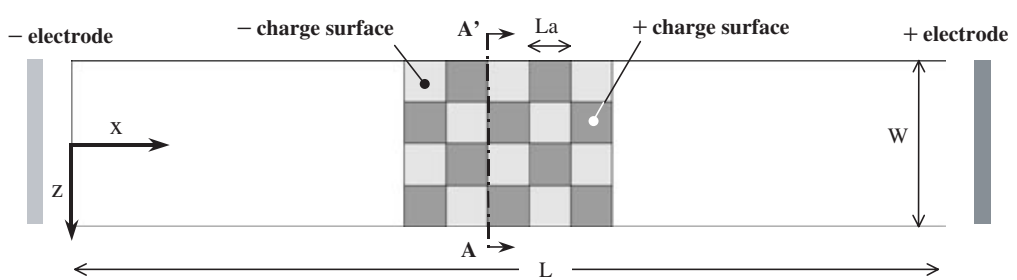

(a)

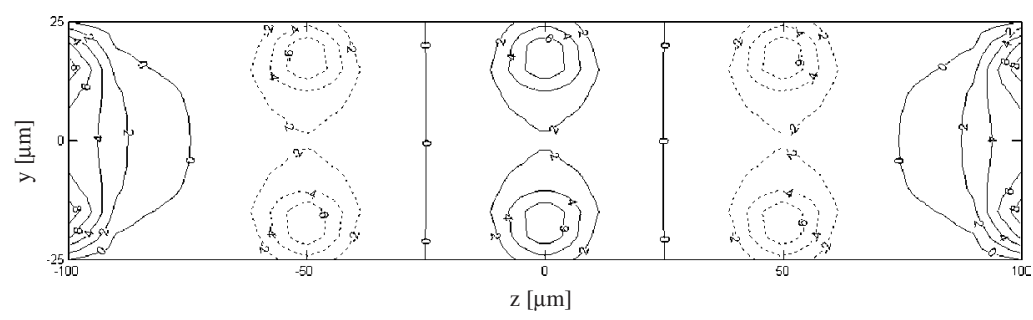

(b)

Figure 13. Streamwise vortical eddies: $(a)$ planar view of the top surface-charge pattern; $(b)$ end view of the corresponding contours of constant streamwise vorticity, $\Omega_{x}(y, z)$, at channel cross-section A-A' with $L_{a}=W_{a}=50 \mu \mathrm{m}$. 
demonstrate the potential of this new approach for microscale mixing applications [18].

\section{Conclusions}

Taking advantage of a new surface-chemistry technology to photolithographically define the surface charge to be negative, neutral or positive, a wide range of liquid flow patterns in microchannels under identical electric field can be created. Design methodologies to generate either in-plane or out-ofplane single and periodic train of vortices have been presented; both co-rotating and counter-rotating vortices are possible. The co-rotating vortices degenerate to simple shear flows at the limit when the area of the neutral zone between the charged regions, either negative or positive, vanishes. Furthermore, the generation of streamwise vorticity has been demonstrated, combining the patterns required for the spanwise and crossstream vorticity, under a streamwise electric field only. Clearly, applying an electric field in other directions and patterning the sidewalls surface charge will enhance the variety of liquid flow patterns that can be produced even further.

\section{Acknowledgments}

This work is supported by the Hong Kong Research Grant Council through RGC grant HKUST 6082/00E and Institute of Integrated Microsystems Grant HKUST I2MS01/02.EG08.

\section{References}

[1] Burn M A, Johnson B N, Brahmasandra S N, Handique K, Webster J R, Krishnan M, Sammarco T S, Man P M, Jones D, Heldsinger D, Mastrangelo C H and Burke D T 1998 An integrated nanoliter DNA analysis device Science 282 484-7

[2] Reyes D R, Iossifidis D, Auroux P A and Manz A 2002 Micrototal analysis systems: 1 . Introduction, theory, and technology Anal. Chem. 74 2623-36

[3] Auroux P A, Iossifidis D, Reyes D R and Manz A 2002 Micrototal Analysis Systems: 2. Anaytical standard operations and application Anal. Chem. 74 2637-52
[4] Bousse L et al 2000 Electrokinetically controlled microfluidic analysis systems Annu. Rev. Biophys. Biomol. Struct. 29 155-81

[5] Hunter R J 1981 Zeta Potential in Colloid Science: Principles and Applications (London: Academic)

[6] Yang C and Li D 1998 Analysis of electrokinetic effects on the liquid flow in rectangular microchannels Colloids Surf. A 143 339-53

[7] Ajdari A 2002 Transverse electrokinetic and microfluidic effects in micropatterned channels: lubrication analysis for slab geometries Phys. Rev. E 65 016301-9

[8] Patankar N A and Hu H H 1998 Numerical simulation of electro-osmotic flow Anal. Chem. 70 1870-81

[9] Ermakov S V, Jacobson S C and Ramsey J M 1998 Computer simulation of electrokinetic transport in microfabricated channel structures Anal. Chem. 70 4494-504

[10] Bianchi F, Ferrigno R and Girault H H 2000 Finite element simulation of an electro-osmotic-driven flow division at a t-junction of microscale dimension Anal. Chem. 72 1987-93

[11] Mitchell M J, Qiao R and Aluru N R 2000 Meshless analysis of steady-state electro-osmotic transport $J$. Microelectromech. Syst. 9 435-49

[12] Dutta P, Beskok A and Warburton T C 2002 Electro-osmotic flow control in complex microgeometries J. Microelectromech. Sys. 11 36-44

[13] Stroock A D, Weck M, Chiu D T, Huck W T S, Kenis P J A, Ismagilov R F and Whitesides G M 2000 Patterning electro-osmotic flow with patterned surface charge Phys. Rev. Lett. 84 3314-7

[14] Hau W L W, Trau D W, Sucher N J, Wong M and Zohar Y 2003 Surface-chemistry technology for microfluidics J. Micromech. Microeng. 13 272-8

[15] Bard A J and Faulkner L R 1980 Electrochemical Methods: Fundamentals and Applications (New York: Wiley)

[16] Hau W L W, Lee L M, Lee Y K, Wong M and Zohar Y 2003 Experimental investigation of electrokinetically generated in-plane vorticity in a microchannel Transducers'03: Proc. 12th Int. Conf. on Solid-State Sensors, Actuators and Microsystems pp 651-4

[17] Hau W L W, Trau D W, Sucher N J, Wong M and Zohar Y 2002 Micro flow patterns on demand using surfacechemistry technology MEMS'02: Proc. 15th Int. Conf. Micro Electro Mechanical Systems pp 475-8

[18] Hau W L W, Lee L M, Lee Y K and Zohar Y 2003 Electrically-driven vortical motion for mixing of liquids in a microchannel to be presented at microTAS'03: 7th Int. Conf. on Miniaturized Chemical and BioChemical Analysis Systems (Squaw Valley, 5-9 Oct, 2003) 